\title{
Surface structure of liquid metals and the effect of capillary waves: X-ray studies on liquid indium
}

\author{
H. Tostmann \\ Division of Engineering and Applied Sciences, Harvard University, Cambridge, Massachusetts 02138 \\ E. DiMasi \\ Department of Physics, Brookhaven National Laboratory, Upton, New York 11973-5000 \\ P. S. Pershan \\ Division of Engineering and Applied Sciences, Harvard University, Cambridge, Massachusetts 02138 \\ B. M. Ocko \\ Department of Physics, Brookhaven National Laboratory, Upton, New York 11973-5000 \\ O. G. Shpyrko \\ Division of Engineering and Applied Sciences, Harvard University, Cambridge, Massachusetts 02138 \\ M. Deutsch \\ Department of Physics, Bar-Ilan University, Ramat-Gan 52900, Israel
}

(Received 30 July 1998)

\begin{abstract}
We report X-ray reflectivity (XR) and small-angle off-specular diffuse-scattering (DS) measurements from the surface of liquid indium close to its melting point of $156{ }^{\circ} \mathrm{C}$. From the XR measurements we extract the surface structure factor convolved with fluctuations in the height of the liquid surface. We present a model to describe DS that takes into account the surface structure factor, thermally excited capillary waves, and the experimental resolution. The experimentally determined DS follows this model with no adjustable parameters, allowing the surface structure factor to be deconvolved from the thermally excited height fluctuations. The resulting local electron-density profile displays exponentially decaying surface-induced layering similar to that previously reported for $\mathrm{Ga}$ and $\mathrm{Hg}$. We compare the details of the local electron-density profiles of liquid In, which is a nearly free-electron metal, and liquid $\mathrm{Ga}$, which is considerably more covalent and shows directional bonding in the melt. The oscillatory density profiles have comparable amplitudes in both metals, but surface layering decays over a length scale of $3.5 \pm 0.6 \AA$ for In and $5.5 \pm 0.4 \AA$ for Ga. Upon controlled exposure to oxygen, no oxide monolayer is formed on the liquid In surface, unlike the passivating film formed on liquid gallium. [S0163-1829(99)11701-6]
\end{abstract}

\section{INTRODUCTION}

The structure of the free surface of a liquid metal is fundamentally different from that of a dielectric liquid such as water or argon. For dielectric liquids, the interatomic or intermolecular potential is long-ranged and the nature of the interactions does not change significantly across the liquidvapor interface. Theoretical modeling and molecular dynamics studies result in an interfacial density profile that varies monotonically from the bulk liquid density to the bulk vapor density. ${ }^{1}$ This can be contrasted with results of computer simulations of dielectric liquid noble gases in contact with their solid phase. ${ }^{2}$ There, the hard wall provided by the solid phase is shown to induce surface layering in the liquid with a well defined lamellar structure.

In liquid metals, the potential energy function changes drastically from a screened short-ranged Coulomb potential in the bulk liquid phase where the conduction electrons are delocalized to a long-ranged van der Waals type potential in the vapor phase where all electrons are localized. ${ }^{3}$ In this case, density functional calculations ${ }^{4,5}$ and computer simulations ${ }^{6}$ indicate an oscillatory surface-normal density profile. This surface-induced layering can be attributed to the fact that strong Coulomb interactions between the abruptly truncated conduction electrons at the surface and the ion cores impose a constraint ordering the surface in a well defined lamellar structure. This constraint can be thought of as an effective hard wall ionic potential at the surface where the conduction electron density abruptly terminates.

The existence of surface layering in liquid metals has only recently been verified unambiguously by experiment, first for liquid $\mathrm{Hg}$ (Ref. 7) and then for liquid Ga. ${ }^{8}$ Comparison of the temperature dependence of this layering, however, reveals qualitative differences between the two metals. ${ }^{9,10}$ This observation indicates that details of the interactions such as the degree of covalency in the metal may affect the surface structure.

For crystalline metals that can be described by weak pseudopotentials, electron-ion scattering is relatively weak and the nonlocalized or itinerant electrons can be treated as if they were essentially free. ${ }^{11}$ Alkali metals with their nearly parabolic energy bands are the prime example of this class of 
nearly free-electron (NFE)-like metals. In analogy with this, the term NFE metals applies to liquid metals whose conduction electrons have similar itinerant character and are only weakly perturbated by a small ionic pseudopotential. ${ }^{12} \mathrm{NFE}$ liquid metals can be contrasted with those whose conduction electrons are partially localized in covalent bonds. ${ }^{13}$ Evidence for such covalent character in liquid metals may be obtained from measurements of the optical properties as well as from the bulk liquid structure factor. ${ }^{14}$

In fact, both liquid $\mathrm{Ga}$ and $\mathrm{Hg}$ show substantial deviations from NFE behavior. In liquid $\mathrm{Ga}$, a shoulder in the bulk liquid structure factor indicates the presence of directional bonding in the melt. ${ }^{15-18}$ Liquid $\mathrm{Hg}$ displays a pronounced asymmetry in the first peak of the bulk liquid structure factor, and the optical constants of liquid $\mathrm{Hg}$ deviate markedly from the predictions of the Drude theory for free electrons, ${ }^{19}$ which, for example, adequately describes the optical constants of the alkali metals. ${ }^{20,21}$ In order to understand which aspects of surface layering may be affected by deviations from NFE character, it is necessary to investigate liquid metals with less tendency towards covalent bonding. The alkali metals would be ideal candidates in this regard. Unfortunately, the very low surface tension of liquid alkali metals will result in a large thermally induced surface roughness, making it extremely difficult to observe the surface layering experimentally. This is exacerbated by the fact that alkali metals are very reactive in air but cannot be investigated under ultrahigh vacuum (UHV) conditions due to their high vapor pressure. Liquid indium, which has a high surface tension, reasonably low melting point, and low vapor pressure does not suffer from these shortcomings. Moreover, it is considered to be NFE-like for the following reasons. First, the first peak in the bulk structure factor of liquid In is highly symmetric, indicating the absence of significant orientational bonding in the melt. ${ }^{22}$ Second, quantitative analysis of the shape of the bulk liquid structure factor yields a twelvefold coordination with an interatomic distance that decreases with increasing temperature. ${ }^{23}$ This is precisely what is expected for a liquid that can be described by an ideal hard sphere model. Third, the measured optical properties of liquid In agree with the Drude free-electron theory over a large range of wave vectors. ${ }^{24}$ Even more interesting, the optical constants of mixtures of liquid $\mathrm{Hg}$ and In differ appreciably from free electron behavior for $\mathrm{Hg}$ rich compositions, but gradually approach free-electron behavior with increasing In content. ${ }^{25}$ A main objective of this study is to compare the surface structure of the more NFE-like liquid metal In to that of the more covalent liquid Ga.

This fundamental interest in the relationship between the surface structure and electronic properties of liquid metals bears directly on the topic of surface reactions, which is of considerable practical importance. This is especially true for liquids, where the atoms are mobile, and reactions at the surface can directly affect alloying, phase formation, and other properties of the bulk. For this reason, surfaces and reactions at surfaces play a critical role in process and extractive metallurgy. ${ }^{26}$ We previously demonstrated that on exposure to oxygen the surface of liquid Ga becomes coated with a uniform $5 \AA$ thick passivating oxide film that protects the underlying bulk phase from further oxidation. ${ }^{27}$ We will show below that the oxidation behavior of liquid In is fundamentally different from that of $\mathrm{Ga}$.

\section{EXPERIMENTAL DETAILS}

The experiments reported here were primarily performed at the beamline X25 at the National Synchrotron Light Source (NSLS). Following a toroidal focussing mirror, a Ge (220) crystal was used to select the $\mathrm{x}$-ray wavelength $\lambda$ $=0.653 \AA$ and to tilt the beam downward onto the horizontal liquid metal surface. The geometry of this liquid spectrometer has been described elsewhere. ${ }^{28}$ Additional data were acquired at beamline X22B at the NSLS, using a similar scattering geometry and an x-ray wavelength of $1.24 \AA$. The size of the $\mathrm{x}$-ray beam striking the liquid metal surface was determined by $1 \mathrm{~mm}$ (horizontal) $\times 0.1 \mathrm{~mm}$ (vertical) slits downstream of the monochromator and the measured signal was normalized to the incoming beam intensity.

The experimental resolution, determined primarily by slits in front of the detector, was varied for different experimental configurations. Specular reflectivity was primarily measured using a conventional NaI scintillation detector. Typical slit settings for measuring this specular reflectivity at X25 were $4 \mathrm{~mm}(\mathrm{~h}) \times 4 \mathrm{~mm}(\mathrm{v})$. At $600 \mathrm{~mm}$ from the sample this yields a $q_{z}$ resolution of $0.06 \AA^{-1}$. For comparable resolution at the longer wavelength at $\mathrm{X} 22 \mathrm{~B}$, a vertical detector slit of 8 $\mathrm{mm}$ was used. Small angle off-specular surface diffuse scattering was measured using a position sensitive detector (PSD) aligned within the reflection plane. ${ }^{29}$ For a given incident angle, the PSD detects both the specular reflection and off-specular diffuse intensity in the reflection plane up to $\approx 0.5^{\circ}$ away from the specular peak. We used a coarse vertical resolution for the PSD in the multichannel mode (64 channels on $20 \mathrm{~mm}$ ). Away from the specular peak, where the intensity varies slowly with position, data from neighboring channels were averaged together for improved statistics. Counting all photons hitting the detector, and disregarding the positional information, the PSD can be used as a conventional counter. In this "single channel" mode the slit settings were $20 \mathrm{~mm}$ vertically and $3 \mathrm{~mm}$ horizontally, corresponding to a $q_{z}$ resolution of $0.32 \AA^{-1}$. For all experimental arrangements, the signal originating at the surface was separated from the bulk diffuse background by subtracting intensities measured with the detector moved approximately $\pm 0.4^{\circ}$ (corresponding to $\approx 0.67 \AA^{-1}$ ) transverse to the reflection plane. ${ }^{28}$

The linearity of the PSD was checked by comparison of diffuse scans taken with the PSD to diffuse data taken by moving the scintillation detector within the reflection plane, and by measuring the specular reflectivity with the stationary PSD in a single-channel mode. As we will discuss below, once scaled for the different resolution, the different types of scans perfectly overlap each other.

Indium of $99.9995 \%$ purity was contained in a Molybdenum pan within a UHV chamber to maintain a clean surface. ${ }^{30}$ Details of the UHV chamber design can be found elsewhere. ${ }^{28}$ The sample was heated above the melting point of liquid In to $170 \pm 5{ }^{\circ} \mathrm{C}$. After a three-day bakeout, the pressure was $8 \times 10^{-10}$ Torr, with a partial oxygen pressure $<8 \times 10^{-11}$, for which the formation time for an oxide monolayer is calculated to be several days. ${ }^{31}$ Although 


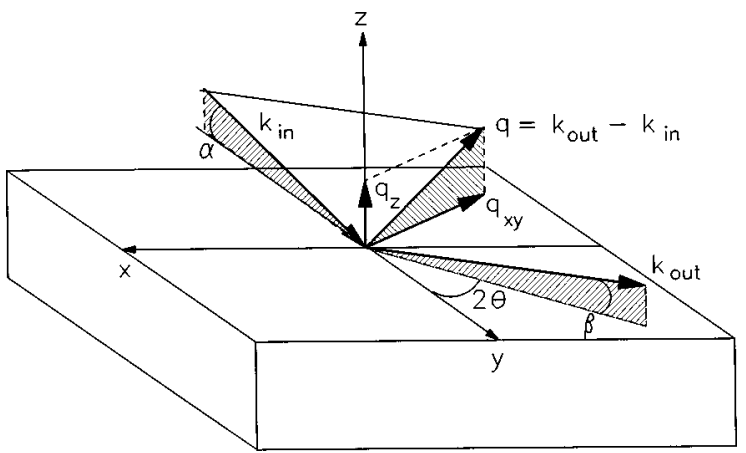

FIG. 1. Sketch of the geometry of x-ray scattering from the liquid surface with $\alpha$ and $\beta$ denoting incoming and outgoing angle, the incoming and outgoing wave vector $\vec{k}_{\text {in }}$ and $\vec{k}_{\text {out }}$, respectively, and the azimuthal angle $2 \theta$. The momentum transfer $\vec{q}$ has an inplane component $q_{x y}$ and a surface-normal component $q_{z}$.

patches of oxide were present on the In ingot when it was introduced into the chamber, this oxide was removed by argon ion sputtering $(2.0 \mathrm{keV}, 30 \mu \mathrm{A}$ ion current) at an $\mathrm{Ar}$ partial pressure of about $5 \times 10^{-5}$ Torr. The impact of the ion beam induced mass flow along the surface that transported the oxide patches towards the ion beam, thus cleaning the entire liquid-vacuum interface and not only the area hit directly by the $\mathrm{Ar}^{+}$ions. ${ }^{32}$ Data presented here were always taken within one to two hours of sputter cleaning the sample. Since data acquired without sputtering but within a few hours reproduced each other, and because of the unique growth properties of oxide on liquid In which will be presented below, we are confident that the measured scattering is intrinsic to a clean liquid indium surface.

A major improvement over experiments described previously is the incorporation of active vibration isolation. Previous measurements eliminated mechanically induced surface waves by utilizing the viscous drag at the bottom of thin samples. These samples had curved surfaces, requiring a time consuming technique to measure the reflectivity ${ }^{28}$ and making it very difficult to measure at grazing incidence, important for the detection of in-plane ordering. In this work, the rigid UHV chamber and ion pump assembly were mounted on an active vibration isolation unit, ${ }^{33}$ similar to that used previously in our studies of liquid $\mathrm{Hg}^{7}{ }^{7}$ With this system we were able to use a sample pan $5 \mathrm{~mm}$ deep and 60 $\mathrm{mm}$ in diameter, resulting in flat samples where the angular deviation of the surface normal from the vertical across the surface was negligible compared to the critical angle for total external reflection of $\mathrm{x}$ rays.

\section{THEORY}

The scattering geometry is given in Fig. 1, defining the liquid surface to be lying in the $x-y$ plane, with $\mathrm{x}$ rays incident at an angle $\alpha$ and collected by the detector at an elevation angle $\beta$ and azimuthal angle $2 \theta$. The momentum transfer $\vec{q}$ can be decomposed into surface normal, $q_{z}$, and surface parallel, $q_{x y}$, components given by

$$
q_{z}=\frac{2 \pi}{\lambda}(\sin \beta+\sin \alpha)
$$

$$
q_{x y}=\frac{2 \pi}{\lambda} \sqrt{\cos ^{2} \alpha+\cos ^{2} \beta-2 \cos \alpha \cos \beta \cos 2 \theta} .
$$

In the following, we develop a formula for the differential cross section $d \sigma / d \Omega$ for $\mathrm{x}$-ray scattering from a rough and structured liquid surface. The general expression for the differential cross section for x-ray scattering from a threedimensional electron distribution $\rho(\vec{r})$ at a given location $\vec{r}$ can be expressed in terms of the electron density correlation function $\left\langle\rho(\vec{r}) \rho\left(\vec{r}^{\prime}\right)\right\rangle=\left\langle\rho\left(\vec{r}-\vec{r}^{\prime}\right) \rho(0)\right\rangle::^{34}$

$$
\begin{aligned}
\frac{d \sigma}{d \Omega}= & V\left(\frac{e^{2}}{m c^{2}}\right)^{2} \int d^{3}\left(\vec{r}-\vec{r}^{\prime}\right)\left\langle\rho\left(\vec{r}-\vec{r}^{\prime}\right) \rho(0)\right\rangle \\
& \times \exp \left[l \vec{q} \cdot\left(\vec{r}-\vec{r}^{\prime}\right)\right],
\end{aligned}
$$

where $V$ is the illuminated volume. If the density distribution is homogeneous within the $x-y$ plane but inhomogeneous normal to the surface, the density correlation function depends on the different positions $\vec{r}_{x y}-\vec{r}_{x y}^{\prime}$ on the surface and the distances $z$ and $z^{\prime}$ from the surface. For an x-ray beam of a cross sectional area $A_{0}$ incident on the surface at an angle $\alpha$ relative to the $x-y$ plane, the illuminated area is $A_{0} / \sin (\alpha)$ and the differential cross section can be written: ${ }^{35}$

$$
\begin{aligned}
\frac{d \sigma}{d \Omega}= & \frac{A_{0}}{\sin (\alpha)}\left(\frac{e^{2}}{m c^{2}}\right)^{2} \int d z d z^{\prime} d^{2} \vec{r}_{x y}\left\langle\rho\left(\vec{r}_{x y}, z\right) \rho\left(0, z^{\prime}\right)\right\rangle \\
& \times \exp \left[\imath q_{z}\left(z-z^{\prime}\right)+\imath \vec{q}_{x y} \cdot \vec{r}_{x y}\right] .
\end{aligned}
$$

For a homogeneous liquid that is sufficiently far away from any critical region, the only long wavelength excitations that give rise to significant scattering are thermally excited surface capillary waves. In this case, there is some length $\xi$ such that for $\left|\vec{r}_{x y}\right|>\xi$ the density density correlation function can be expressed in terms of a density profile $\widetilde{\rho}\left[z-h\left(\vec{r}_{x y}\right)\right]$ defined relative to the local position of the liquid surface $h\left(\vec{r}_{x y}\right)$ and capillary wave induced relative variations of the height, $h\left(\vec{r}_{x y}\right)-h\left(\vec{r}_{x y}^{\prime}\right){ }^{36}$

$$
\left\langle\rho\left(\vec{r}_{x y}, z\right) \rho\left(0, z^{\prime}\right)\right\rangle \stackrel{\left|\vec{r}_{x y}\right|>\xi}{\longrightarrow} \widetilde{\rho}\left(z-\left[h\left(\vec{r}_{x y}\right)-h(0)\right]\right) \widetilde{\rho}\left(z^{\prime}\right) .
$$

Defining $\delta \rho\left(\vec{r}_{x y}, z\right)=\rho\left(\vec{r}_{x y}, z\right)-\widetilde{\rho}\left(z-h\left(\vec{r}_{x y}\right)\right)$, the differential cross section from a liquid surface can now be written:

$$
\begin{aligned}
\frac{d \sigma}{d \Omega}= & \frac{A_{0}}{\sin (\alpha)}\left(\frac{e^{2}}{m c^{2}}\right)^{2} \int d z d z^{\prime} d^{2} \vec{r}_{x y} \\
& \times \exp \left[l q_{z}\left(z-z^{\prime}\right)+\imath \vec{q}_{x y} \cdot \vec{r}_{x y}\right]\left\{\widetilde { \rho } \left(z-\left[h\left(\vec{r}_{x y}\right)\right.\right.\right. \\
& \left.-h(0)]) \widetilde{\rho}\left(z^{\prime}\right)+\left\langle\delta \rho\left(\vec{r}_{x y}, z\right) \delta \rho\left(0, z^{\prime}\right)\right\rangle\right\} .
\end{aligned}
$$

The second term in the integrand is only nonvanishing for regions $\left|\vec{r}_{x y}\right| \leqslant \xi$. This term gives rise to both the bulk diffuse scattering and surface scattering at momentum transfers having large $q_{x y}$ components. ${ }^{35}$ The effect of this term can be separated experimentally from the specular reflection and may be omitted in the following discussion of scattering close to the specular condition. A change of variables yields 


$$
\begin{aligned}
\frac{d \sigma}{d \Omega} \approx & \frac{1}{16 \pi^{2}}\left(\frac{q_{c}}{2}\right)^{4} \frac{A_{0}}{\sin (\alpha)} \frac{\left|\Phi\left(q_{z}\right)\right|^{2}}{q_{z}^{2}} \\
& \times \int_{\left|\vec{r}_{x y}\right|>\xi} d^{2} \vec{r}_{x y}\left\langle\exp \left\{l q_{z} h\left(\vec{r}_{x y}\right)\right\}\right\rangle \exp \left[\imath \vec{q}_{x y} \cdot \vec{r}_{x y}\right],
\end{aligned}
$$

where $q_{c}$ is the critical angle for total external reflection of $\mathrm{x}$ rays and the surface structure factor

$$
\begin{aligned}
\Phi\left(q_{z}\right) & =\frac{1}{\rho_{\infty}} \int d z \frac{d \widetilde{\rho}(z)}{d z} \exp \left(\iota q_{z} z\right) \\
& =\frac{-\imath q_{z}}{\rho_{\infty}} \int d z \widetilde{\rho}(z) \exp \left(\imath q_{z} z\right)
\end{aligned}
$$

is the Fourier transform of the local or intrinsic density profile $\widetilde{\rho}(z)$, independent of $\vec{r}_{x y}$. In this equation, $\rho_{\infty}$ is the bulk electron density.

For the case of thermally excited capillary waves on a liquid surface, the height fluctuations can be characterized by their statistical average $\left\langle\left|h\left(\vec{r}_{x y}\right)\right|^{2}\right\rangle{ }^{35}$ Sinha et al. have shown that integration over these height fluctuations yields the following dependence of the scattering on $q_{x y}$ and $q_{z}:{ }^{37}$

$$
\int_{\left|\vec{r}_{x y}\right|>\xi} d^{2} \vec{r}_{x y}\left\langle\exp \left\{\imath q_{z} h\left(\vec{r}_{x y}\right)\right\}\right\rangle \exp \left[\imath \vec{q}_{x y} \cdot \vec{r}_{x y}\right]=\frac{C}{q_{x y}^{2-\eta}}
$$

with $C$ to be determined and

$$
\eta=\frac{k_{B} T}{2 \pi \gamma} q_{z}^{2} .
$$

The value of $C$ can be determined by integration over all surface capillary modes having wave vectors smaller than the upper wave vector cutoff $q_{\max } \equiv \pi / \xi$ :

$$
\begin{aligned}
C= & \int_{\left|\vec{q}_{x y}\right|<\pi / \xi} d^{2} \vec{q}_{x y} \int_{\left|\vec{r}_{x y}\right|>\xi} d^{2} \vec{r}_{x y} \\
& \times\left\langle\exp \left\{l q_{z} h\left(\vec{r}_{x y}\right)\right\}\right\rangle \exp \left[\imath \vec{q}_{x y} \cdot \vec{r}_{x y}\right] \\
\approx & 4 \pi^{2} \lim _{\vec{r}_{x y} \rightarrow 0}\left\langle\exp \left\{l q_{z} h\left(\vec{r}_{x y}\right)\right\}\right\rangle=4 \pi^{2} .
\end{aligned}
$$

Therefore, the properly normalized differential cross section for scattering of $\mathrm{x}$ rays from a liquid surface is

$$
\frac{d \sigma}{d \Omega}=\frac{A_{0}}{\sin \alpha}\left(\frac{q_{c}}{2}\right)^{4} \frac{k_{B} T}{16 \pi^{2} \gamma}\left|\Phi\left(q_{z}\right)\right|^{2} \frac{1}{q_{x y}^{2}}\left(\frac{q_{x y}}{q_{\max }}\right)^{\eta} .
$$

The intensity measured at a specific scattering vector $\vec{q}$ is obtained by integrating Eq. (10) over the solid angle $d \Omega$ defined by the detector acceptance of the experiment. For the specular reflection, $q_{x y}=0(\alpha=\beta$ and $2 \theta=0)$, the integral is centered at $q_{x y}=0$. The projection of the detector resolution onto the $x-y$ plane is rectangular ${ }^{35}$ and the above-mentioned integration has to be done numerically. However, an analytical formula can be obtained for the specular reflectivity if the detector resolution is assumed to be a circle of radius $q_{\text {res }}$, independent of $q_{z}:^{28}$

$$
\frac{R\left(q_{z}\right)}{R_{f}\left(q_{z}\right)}=\left|\Phi\left(q_{z}\right)\right|^{2}\left(\frac{q_{\mathrm{res}}}{q_{\mathrm{max}}}\right)^{\eta}=\left|\Phi\left(q_{z}\right)\right|^{2} \exp \left[-\sigma_{\mathrm{cw}}^{2} q_{z}^{2}\right],
$$

where

$$
R_{f}\left(q_{z}\right)=\left|\frac{q_{z}-\sqrt{q_{z}^{2}-q_{c}^{2}}}{q_{z}+\sqrt{q_{z}^{2}-q_{c}^{2}}}\right|^{2} \approx\left(\frac{q_{c}}{2 q_{z}}\right)^{4} \text { for } q_{z} \gtrsim 5 q_{c}
$$

is the Fresnel reflectivity from classical optics for a flat $\left[h\left(\vec{r}_{x y}\right)=h(0)\right.$ for all $\left.h\left(\vec{r}_{x y}\right)\right]$ and structureless $\left(\left|\Phi\left(q_{z}\right)\right|^{2}\right.$ $=1)$ surface. The quantity $\sigma_{\mathrm{cw}}$ denotes the capillary wave roughness:

$$
\sigma_{\mathrm{cw}}^{2}=\frac{k_{B} T}{2 \pi \gamma} \ln \left(\frac{q_{\text {res }}}{q_{\mathrm{max}}}\right) .
$$

In the experiments presented here, the measured specular reflectivity was analyzed by numerical integration of the theoretical model over the rectangular resolution defined by the detector slits. In practice, the specular reflection is measured with coarse resolution and the results based on Eq. (11) fall within $5 \%$ of the exact values obtained from integrating over Eq. (10). On the other hand, the off specular diffuse scattering is measured with much finer resolution and near the specular peak, $\vec{q}_{x y}=0$, it has to be analyzed by numerical integration of Eq. (10) over the slit defined resolution. In addition, numerical integration of Eq. (10) is necessary to obtain the correct ratio of specular reflectivity to off-specular diffuse scattering.

In Eq. (7) we have introduced a local structure factor which is the Fourier transform of the local or intrinsic density profile. An alternative representation is to define a macroscopically averaged density profile $\langle\rho(z)\rangle_{T}$ such that

$$
\frac{1}{\rho_{\infty}} \int d z \frac{\langle\rho(z)\rangle_{T}}{d z} \exp \left(\iota q_{z} z\right)=\Phi\left(q_{z}\right) \exp \left(-1 / 2 \sigma_{\mathrm{cw}}^{2} q_{z}^{2}\right) .
$$

This macroscopically averaged density profile, $\langle\rho(z)\rangle_{T}$, can be shown to be equal to the convolution of the local density profile, $\widetilde{\rho}(z)$, with the associated Gaussian distribution of height fluctuations characterized by

$$
\sigma_{\mathrm{cw}}^{2}=\left\langle\left|h\left(\vec{r}_{x y}\right)\right|^{2}\right\rangle=\frac{k_{B} T}{2 \pi \gamma} \ln \left(\frac{1}{\left|\vec{r}_{x y}\right| q_{\max }}\right) .
$$

Unfortunately, it is difficult to extract a precisely defined $\langle\rho(z)\rangle_{T}$ from a single reflectivity measurement because of the above-mentioned problem of the variation of the resolution with $q_{z}$.

A second difficulty with reflectivity measurements, even when $\left|\Phi\left(q_{z}\right)\right|^{2}$ can be separated from the thermal effects, is that the direct inversion of $\left|\Phi\left(q_{z}\right)\right|^{2}$ in order to obtain $\widetilde{\rho}(z)$ is not possible because the phase information necessary to perform the Fourier transform in Eq. (7) is lost in any intensity measurement. The common practice to overcome this problem is to assume a model for the density profile, which is then Fourier transformed and fitted to the experimentally determined structure factor. In this study, the liquid metal is modeled by layers of different electron densities parallel to the surface. The layers represent planes of atoms, separated by a distance $d$, and having increasing width the further they 


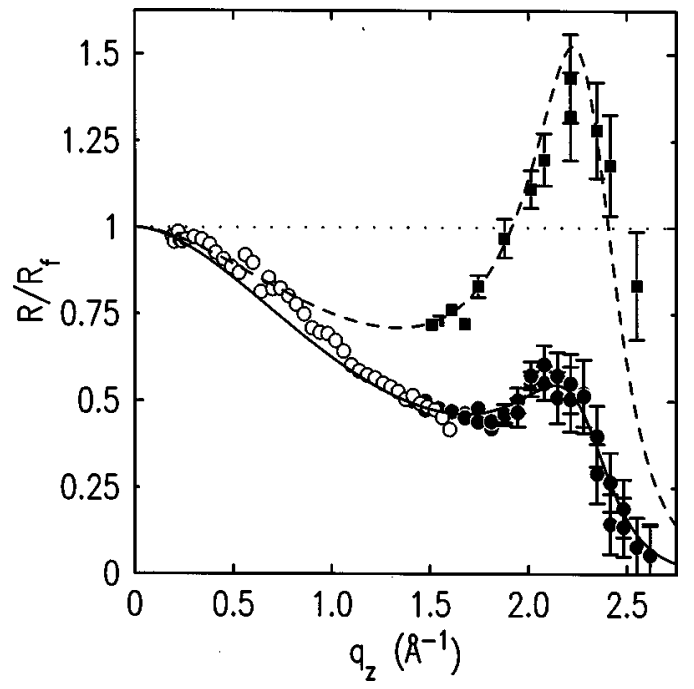

FIG. 2. Specular $\mathrm{x}$-ray reflectivity for liquid indium at $170{ }^{\circ} \mathrm{C}$ taken with vertical detector resolutions of $0.06 \AA^{-1}$ (scintillation detector; open circles: X22B, closed circles: X25) and $0.32 \AA^{-1}$ (PSD in single channel mode; squares: $\mathrm{X} 25$ ). The reflectivity is normalized to the Fresnel reflectivity, $R_{f}$, of a flat In surface. Solid lines are fits as described in the text. Data from X22B was not incorporated in any fits.

are below the surface. Mathematically, the electron density is described by a semi-infinite sum of Gaussians, normalized to the bulk density $\rho_{\infty}$ :

$$
\widetilde{\rho}(z)=\rho_{\infty} \sum_{n=0}^{\infty} \frac{d / \sigma_{n}}{\sqrt{2 \pi}} \exp \left[-(z-n d)^{2} / 2 \sigma_{n}^{2}\right] \otimes F_{\mathrm{In}}(z)
$$

Here $\otimes$ denotes convolution, $F_{\mathrm{In}}(z)$ is the atomic x-ray scattering form factor of In, and $\sigma_{n}^{2}=n \bar{\sigma}^{2}+\sigma_{0}^{2}$, where $\bar{\sigma}$ and $\sigma_{0}$ are constants. This form for $\sigma_{n}$ produces a quadratic increase in the Gaussian width with distance $z$ below the surface, so that the parameter $\bar{\sigma}$ is related to the decay length for surface layering and the model density approaches the bulk density $\rho_{\infty}$ for $\sigma_{n} \gg d$. An advantage of using Gaussian functions to model $\widetilde{\rho}(z)$ is that an expression for $\langle\rho(z)\rangle_{T}$ is obtained from Eq. (13) by replacing $\sigma_{n}$ with $\sigma_{n}^{T}$ and defining $\left(\sigma_{n}^{T}\right)^{2}$ $=\sigma_{\mathrm{cw}}^{2}+\sigma_{n}^{2}$. Note that the effective width of the individual layers becomes $\left(\sigma_{n}^{T}\right)^{2}$ which explicitly demonstrates the additivity of the different contributions to the surface roughness from capillary modes (for which $q_{x y}<q_{\max }$ ) and short wavelength modes (for which $q_{x y}>q_{\max }$ ) that are incorporated into the model for $\widetilde{\rho}(z)$.

\section{EXPERIMENTAL RESULTS}

The specular reflectivity measured from liquid In at 170 $\pm 5{ }^{\circ} \mathrm{C}$ demonstrates the presence of surface layering. Figure 2 shows the reflectivity as a function of $q_{z}$, normalized to the Fresnel reflectivity of a sharply terminated In surface with a critical angle $q_{c}=0.052 \AA^{-1}$. The circles show the data taken with the scintillation detector, with a $q_{z}$ resolution of $0.06 \AA^{-1}$. Open circles represent the small angle data taken at X22B $(\lambda=1.24 \AA)$ whereas the closed circle data have been taken at X25 $(\lambda=0.65 \AA)$. Filled squares repre- sent data taken with the PSD in a single channel mode, with the vertical resolution coarser by a factor of 5 . The large increase in signal with decreasing resolution immediately demonstrates the significance of the diffuse scattering. The reflectivity at small and intermediate wave vectors falls below the Fresnel value calculated for an ideally flat and abruptly terminated surface. This is due to the roughness of the liquid In surface which scatters photons out of the specular condition. The most prominent feature in these data is the broad peak centered near $2.2 \AA^{-1}$. This peak is due to constructive interference of $\mathrm{x}$ rays from layers ordered parallel to the surface. This very distinctive structural feature of liquid metal surfaces has been observed earlier for $\mathrm{Hg}$ (Ref. 7) and $\mathrm{Ga}^{8}$ The two data sets show the same features, although as a consequence of integrating the diffuse scattering over coarser resolution, the intensity is greater for data taken with the PSD.

The lines in Fig. 2 illustrate fits of the density profile model discussed above to these data. Three surface layering parameters, $d, \bar{\sigma}$, and $\sigma_{0}$, determine the form of $\Phi\left(q_{z}\right)$, which is then used in Eq. (10) along with $T=170{ }^{\circ} \mathrm{C}$ and the experimentally determined value for the surface tension of liquid In at that temperature, $\gamma=0.556 \mathrm{~N} / \mathrm{m} .{ }^{38}$ As discussed above, we assign the short wavelength cutoff for capillary waves, $q_{\text {max }} \approx \pi / \xi$, to be $\sim 1 \AA^{-1}$ where $\xi$ was taken to be the nearest neighbor atomic distance in the bulk melt. ${ }^{39}$ The integral over Eq. (10) is then performed numerically over the known resolution volume. Surface layering parameters obtained from the scintillation detector data are $d=2.69$ $\pm 0.05 \AA, \bar{\sigma}=0.54 \pm 0.06 \AA$, and $\sigma_{0}=0.35 \pm 0.04 \AA$ (solid line in Fig. 2). Essentially the same parameters (with larger error due to poorer statistics) result from a fit of the model to the PSD data (broken line in Fig. 2).

Although the resolution dependence of the reflectivity of liquid metals provides a test of the thermal capillary wave theory prediction, a more rigorous test is the measurement of the spectral density of the off specular diffuse scattering. We have measured diffuse intensity over a $\beta$ range straddling the specular condition. Intensities normalized to the direct beam are shown in Fig. 3 for several choices of $\alpha$. Data at extreme values of $(\beta-\alpha)$ are limited by the intense background due to bulk scattering at large $\beta$ and by the scattering geometry at small $\beta$. The asymmetry of the wings centered around the specular ridge $(\beta-\alpha=0)$ arises from the $\beta$ dependence of both the exponent $\eta$ and the surface structure factor $\Phi\left(q_{z}\right)$.

The solid lines are obtained by calculating the intensity from Eq. (10), integrating numerically over the resolution function and subtracting a similar calculation for the background scan taken out of the reflection plane. The calculation incorporates the surface structure factor $\Phi\left(q_{z}\right)$ determined from the reflectivity measurements along with the other fixed quantities $\left(\gamma, k_{B} T\right.$, and $\left.q_{\text {max }}\right)$, without further adjustable parameters. We find excellent agreement between this model and the experimental data for the entire range studied, including the wings (Fig. 3) and the specular region (inset of Fig. 3). The implications of this agreement will be discussed in the next section.

To study the oxidation properties of liquid indium we exposed the In surface to controlled amounts of oxygen through a bakeable UHV leak valve. During oxidation, macroscopic oxide clumps, large enough to be observed by eye, 


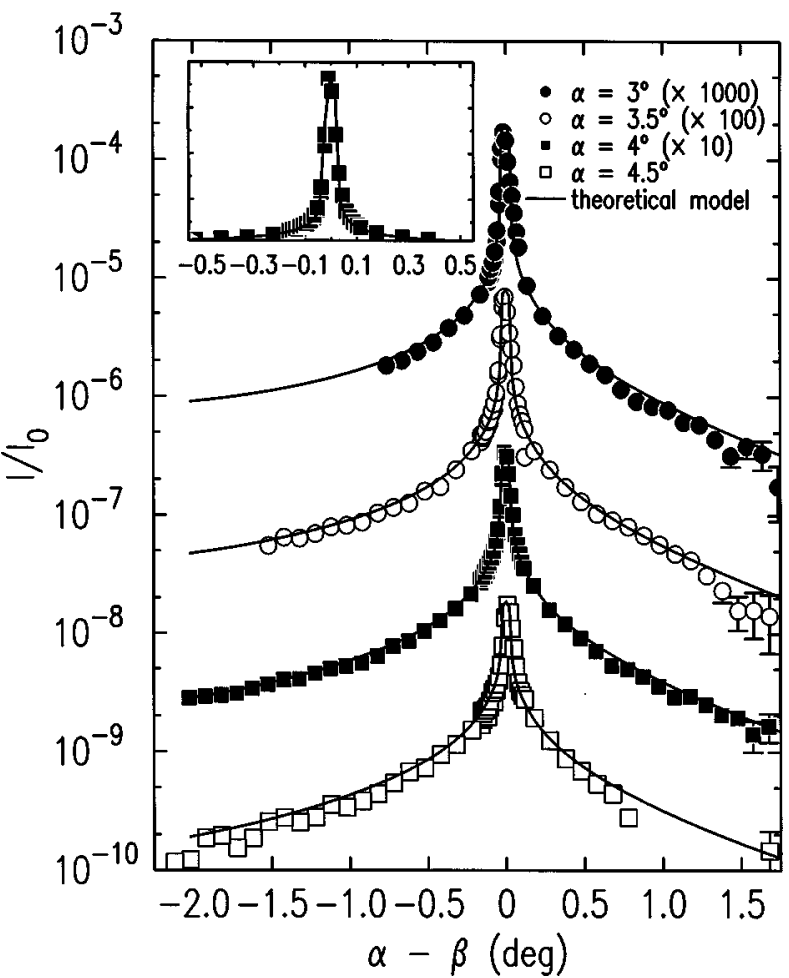

FIG. 3. Diffuse scattering as a function of scattering angle $\beta$ for different fixed incoming angles $\alpha$. Solid lines: Diffuse scattering calculated from the experimentally determined structure factor with no further adjustable parameters. Inset: linear plot emphasizing the fit near the specular peak for $\alpha=4.5^{\circ}$.

formed at the edges of the sample, while the center remained clean. X-ray reflectivity measured during oxidation showed no changes until the floating oxide regions grew large enough to reach the area illuminated by the x-ray beam, at which point the macroscopically rough surface scattered the reflected signal away from the specular condition. This result is in sharp contrast to the formation of the highly uniform, 5 $\AA$ thick, passivating oxide layer we previously observed to form on liquid $\mathrm{Ga}$ under the influence of the same amount of oxygen. $^{27}$

\section{DISCUSSION}

The macroscopic density profile extracted from the reflectivity measurements is resolution dependent since the density oscillations are smeared out by thermally activated capillary waves as described in the Theory section. This smearing depends on the temperature and surface tension and hence also varies for different liquids. The macroscopic density profile directly extracted from the experiment is the averaged density profile $\langle\rho(z)\rangle_{T}$ shown in previous publications. ${ }^{7-9,28}$ In order to compare the intrinsic layering properties in different liquid metals, it is necessary to remove these thermal effects that vary from metal to metal and experiment to experiment and to obtain the intrinsic or local density profile $\widetilde{\rho}(z)$. Previous temperature dependent reflectivity measurements of liquid $\mathrm{Ga}$ around the specular position have shown that the resolution dependent roughness is well described by the capillary wave $(\mathrm{CW})$ prediction. ${ }^{9}$ Similar conclusions were reached from $T$-dependent measurements of liquid

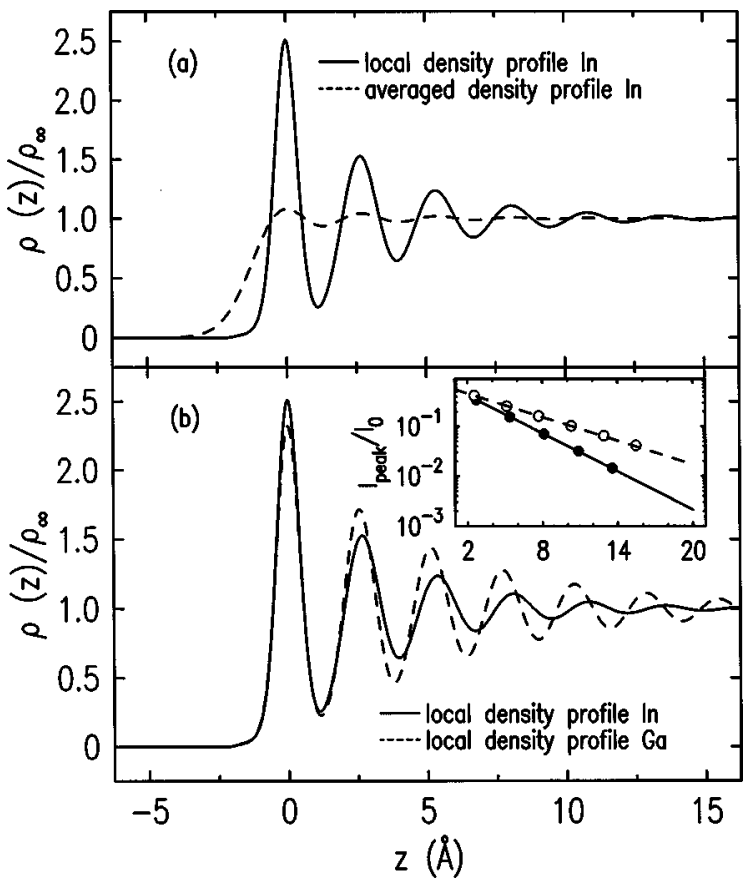

FIG. 4. (a) Comparison of the local real space density profile for liquid indium (-) with the thermally averaged density profile for the same metal $(---)$. The averaged density profile is directly accessible by experiment and has been measured at $170{ }^{\circ} \mathrm{C}$. (b) Real space local density profile for liquid gallium $(---)$ and liquid indium (-) after the removal of thermal broadening. Densities $\widetilde{\rho}(z)$ are normalized to the bulk densities $\rho_{\infty}$ of liquid gallium and indium. Inset: Decay of the amplitude of the surface-normal density profile for liquid Ga (open circles) and liquid In (filled circles). The lines represent the fit of this decay of the surface layering to the form $\exp (-z / l)$ for liquid $\mathrm{Ga}(---)$ and In $(-)$.

paraffins. ${ }^{40}$ Here, we employed a different approach to verify that the $\mathrm{CW}$ prediction holds for liquid In, performing diffuse scattering measurements at a single temperature at different angles of incidence. The magnitude and the angular dependence of the diffuse scattering is in full compliance with the predictions of the capillary wave model without using any adjustable parameters. This is demonstrated by the perfect agreement of the diffuse scattering profiles with the theoretical curves in Fig. 3. This allows us to directly calculate the local density profile $\widetilde{\rho}(z)$ by simply setting $\eta=0$. A comparison between the local density profile $\widetilde{\rho}(z)$ and the temperature averaged density profile $\langle\rho(z)\rangle_{T}$ is made in Fig. 4(a). In this single- $T$ approach the effects of the intrinsic, $T$ $=0$, roughness $\sigma_{0}$, and the cutoff $q_{\max }$ cannot be unambiguously separated, ${ }^{41}$ as was possible for the $T$-dependent measurements in alkanes ${ }^{40}$ and $\mathrm{Ga}^{9}{ }^{9}$ However, this does not affect our main conclusion that the surface roughness, as probed by the DS, is entirely due to thermally activated capillary waves.

The local density profile of liquid In is compared with that of Ga in Fig. 4(b). Profiles derived from the extremal parameters of fits to $R\left(q_{z}\right)$ and from extremal values for temperature, surface tension, and resolution function are indistinguishable on the scale of the figure. The amplitude of the first density peak for In is comparable to that of Ga. This reflects the fact that in the region nearest the surface, the 


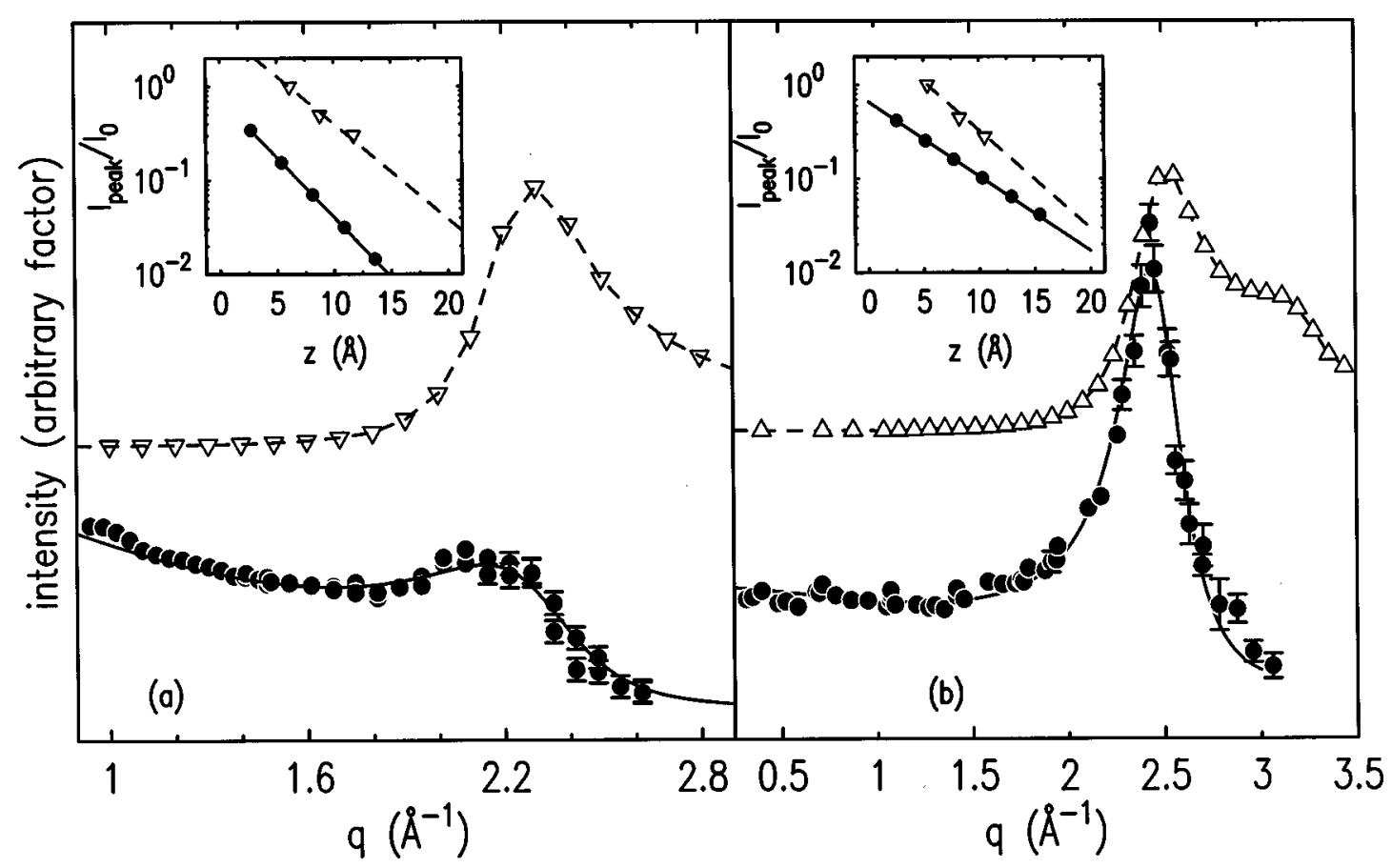

FIG. 5. (a) Bulk $(\triangle)$ and surface $(\bullet)$ structure factors for liquid In. The bulk structure factor data are taken from Orton et al. (Ref. 22). The solid line is a fit of the model explained in the text to the experimentally determined surface structure factor. The broken line is a guide for the eye. The inset compares the decay of the longitudinal surface density oscillations (filled circles; solid line represents fit to an exponential) to the decay of the bulk pair correlation function (open triangles; broken line represents fit to exponential). The coordinate $z$ of the $x$ axis represents the distance from the surface in the case of the decaying surface layering and the radial distance from a reference atom in the bulk liquid in the case of the decaying bulk pair correlation. The data for the bulk pair correlation function are taken from Waseda (Ref. 42). (b) Bulk $(\triangle)$ and surface (O) structure factors for liquid Ga. The bulk structure factor data are taken from Narten et al. (Ref. 15). Solid lines are fits to reflectivity data; broken lines are guides for the eye. The inset compares the decay of the longitudinal surface density oscillations (filled circles; solid line represents fit to exponential) to the decay of the bulk pair correlation function (open triangles; broken line represents fit to exponential). The data for the bulk pair correlation function are taken from Waseda (Ref. 42).

density oscillations are determined primarily by $\sigma_{0}$, which has similar values for the two metals. Further towards the bulk, layering is seen to decay faster for In than for Ga. We quantify this observation by the excellent fit of the peak amplitude of the electron density profile to the form $\exp (-z / l)$. The decay lengths $l=3.5 \AA \pm 0.6$ for In and $l$ $=5.5 \AA \pm 0.4$ for Ga differ by an amount well outside of the experimental error. This is illustrated in the inset of Fig. 4(b), a semilogarithmic plot of the maxima of the density oscillations as a function of distance from the first surface layer. The lines represent the fit of the peak amplitude to the exponential form and the slope represents the decay length.

It is also instructive to compare the surface structure factor measured in the reflectivity experiments to the bulk structure factor measured by standard x-ray or neutron diffraction [Fig. 5(a): indium; Fig. 5(b): gallium]. The widths of the peaks shown are inversely proportional to the decay lengths of the corresponding correlation functions: the pair correlation function $g(r)$ of the bulk and the layered density profile $\widetilde{\rho}(z)$ of the surface. Plotting the amplitudes of $g(r)$ and $\widetilde{\rho}(z)$ on a semilog scale (inset of Fig. 5) shows that they lie on straight lines. This indicates that the correlations decay exponentially, and the decay lengths are obtained as the negative inverses of the slopes. The inset plots show clearly that while the surface and bulk decay lengths are about the same for In, in gallium the surface induced layering decays much more slowly than the bulk pair correlation function. This is consistent with the NFE nature of In. For Ga, by contrast, the tendency towards covalency may disrupt the hard sphere packing and enable surface correlations that differ from those in the bulk.

Another piece of evidence suggesting that directional bonding in the melt might have an effect on surface induced layering stems from the ratio between the surface layer spacing and the bulk nearest neighbor distance. This nearest neighbor distance is taken from the analysis of the pair correlation function which is the Fourier transform of the structure factor depicted in Fig. 5. Due to truncation problems and related subtleties with the data analysis, the bulk nearest neighbor distance cannot be directly inferred from the maximum in the bulk liquid structure factor and we use the nearest neighbor distance given by Iida and Guthrie. ${ }^{38}$ For liquid In, this ratio of surface layer spacing to bulk nearest neighbor distance is $2.69 \AA / 3.14 \AA=0.86$, close to the value of $\sqrt{2 / 3}$ $\approx 0.82$ for the hard sphere packing that would be expected for a NFE liquid metal. For liquid $\mathrm{Ga}$, this ratio is larger, $2.56 \AA / 2.78 \AA=0.92$. In this case, formation of directional bonds, eventually leading to $\mathrm{Ga}_{2}$ dimers, may prevent close packing.

Tomagnini et al. recently considered a possible relationship between surface induced layering in LM and the stability of crystal facets at metal surfaces. Although premelting of crystalline interfaces is quite common in nonmetallic crystals, most metals have at least one close packed face which 
does not premelt, remaining solid up to the melting temperature. ${ }^{43}$ Tomagnini et al. use molecular dynamics simulations to demonstrate that when the period of the surface induced layering at the liquid surface, which they assumed to be $2 \pi / q_{0}$ where $q_{0}$ is the position for the peak in the bulk liquid structure factor, is commensurate with the distance between lattice planes along the normal to the crystal facet, this particular facet is stabilized and resistant to premelting. It would be interesting to correlate the premelting properties of $\mathrm{Ga}$ and In crystals with our results for the period of the surface layering and in particular, to examine if the effect of directional bonding that affects the surface structure in the liquid affects premelting of the solid as well.

Our diffuse scattering measurements show that the roughness of the liquid In surface can be attributed entirely to thermally excited capillary waves. This indicates that there are no other detectable inhomogeneities on the surface, such as might be caused by microscopic oxide patches. This observation is in concert with independent experiments on organic Langmuir monolayers on water. If the monolayer is homogeneous, ${ }^{44,45}$ the DS can be described by Eq. (10) with no adjustable parameters and no excess scattering is observed-just as is the case for metallic liquid In. On the other hand, if the organic monolayer is compressed beyond its elasticity limit and becomes inhomogeneous, Eq. (10) no longer describes the experimentally determined DS, and excess scattering is observed that must originate from sources other than thermally activated capillary waves. ${ }^{44}$ This demonstrates the viability of diffuse scattering as a tool for studying surface inhomogeneities. This is of particular interest for investigations on liquid alloys where-depending on the type of alloy-a rich surface behavior is expected, ranging from concentration fluctuations for alloys displaying surface segregation to critical fluctuations for alloys with a critical consolute point. ${ }^{46}$

Controlled oxidation of the liquid metal surfaces reveals a further striking difference between Ga and In. Although exposure of liquid $\mathrm{Ga}$ to air or to a large amount of oxygen leads to the formation of a macroscopically thick and rough oxide film, exposure to controlled amounts of oxygen under UHV conditions produces a well defined uniform $5 \AA$ thick oxide film. ${ }^{27}$ This film protects the underlying bulk phase to a certain extent from further corrosion, as further dosage at low oxygen pressures $\left(<2 \times 10^{-4}\right.$ Torr $)$ was found to have no effect on the oxide thickness or coverage fraction. This passivating of the surface is rather unusual for solid metals, $\mathrm{Al}$ being prominent among the few examples. Liquid In, un- like $\mathrm{Ga}$, was not found to form a passivating oxide film. Instead, macroscopic clumps of oxide formed which did not wet the clean In surface. This corrosion mechanism is rather common for solid metal surfaces, the oxidation of Fe being the best known example. It is not clear to what extent this fundamental difference in the mechanism for corrosion is related to differences in the structure of the liquid surface and to what extent to the chemical affinity for oxidation of the respective liquid metal.

\section{SUMMARY}

We have measured the x-ray reflectivity and small angle off specular surface diffuse scattering from liquid In at $170{ }^{\circ} \mathrm{C}$. Our results can be quantitatively explained by the convolution of thermally excited surface waves and a temperature independent surface structure factor, corresponding to theoretically predicted surface layering. The absence of excess diffuse scattering beyond that due to thermally excited capillary waves demonstrates that the liquid-vapor interface is homogeneous in the surface-parallel direction. The intrinsic layering profile of liquid In, obtained by removing the capillary wave roughening, is compared to that previously reported for liquid Ga. For Ga, surface layering persists farther into the bulk than is the case for In. This may be attributed to directional bonding in the Ga bond stabilizing surface induced layering over a larger distance than is the case for liquid In. Further evidence suggesting a correlation between the degree of covalency in the melt and the surface structure stems from the observation that the compression of the surface layer spacing relative to the bulk nearest neighbor distance is close to the behavior expected for an ideal hard sphere liquid for In but considerably different for Ga. Controlled oxidation of liquid In results in the formation of a macroscopic rough oxide, unlike the passivating microscopic uniform oxide film that forms on liquid $\mathrm{Ga}$.

\section{ACKNOWLEDGMENTS}

The authors thank Masafumi Fukuto for helpful discussions. This work was supported by the U.S. DOE Grant No. DE-FG02-88-ER45379, the National Science Foundation Grant No. DMR-94-00396, and the U.S.-Israel Binational Science Foundation, Jerusalem. Brookhaven National Laboratory was supported by U.S. DOE Contract No. DE-AC0298CH10886. H.T. acknowledges support from the Deutsche Forschungsgemeinschaft.
${ }^{1}$ F. F. Abraham, Rep. Prog. Phys. 45, 1113 (1982).

${ }^{2}$ G. A. Chapela G. Saville, S. M. Thompson, and J. S. Rowlinson, J. Chem. Soc. Faraday Trans. 73, 1133 (1977).

${ }^{3}$ S. A. Rice et al., Adv. Chem. Phys. 27, 543 (1974).

${ }^{4}$ R. Evans and M. Hasegawa, J. Phys. C 14, 5225 (1981).

${ }^{5}$ M. Iwamatsu and S. K. Lai, J. Phys.: Condens. Matter 4, 6039 (1992)

${ }^{6}$ J. G. Harris, J. Gryko, and S. A. Rice, J. Chem. Phys. 87, 3069 (1987).

${ }^{7}$ O. M. Magnussen, B. M. Ocko, M. J. Regan, K. Penanen, P. S.
Pershan, and M. Deutsch, Phys. Rev. Lett. 74, 4444 (1995).

${ }^{8}$ M. J. Regan, E. H. Kawamoto, S. Lee, P. S. Pershan, N. Maskil, M. Deutsch, O. M. Magnussen, B. M. Ocko, and L. E. Berman, Phys. Rev. Lett. 75, 2498 (1995).

${ }^{9}$ M. J. Regan, P. S. Pershan, O. M. Magnussen, B. M. Ocko, M. Deutsch, and L. Berman, Phys. Rev. B 54, 9730 (1996).

${ }^{10}$ Some pecularities of the $\mathrm{Hg}$ surface structure may be due to the fact that $\mathrm{Hg}$ cannot be studied under ultrahigh vacuum (UHV). In the present paper, we compare the In results to measurements of liquid $\mathrm{Ga}$, both performed under UHV conditions. A detailed discussion of the temperature dependent surface layering of liq- 
uid $\mathrm{Hg}$ will be given elsewhere: E. DiMasi, H. Tostmann, B. M. Ocko, P. S. Pershan, and M. Deutsch (unpublished).

${ }^{11}$ N. W. Ashcroft and N. D. Mermin, Solid State Physics (Harcourt Brace, Orlando, 1976).

${ }^{12}$ J. M. Ziman, Philos. Mag. 6, 1013 (1961).

${ }^{13}$ G. A. de Wijs, G. Pastore, A. Selloni, and W. van der Lugt, Phys. Rev. Lett. 75, 4480 (1995).

${ }^{14}$ N. H. March, Liquid Metals (Cambridge University Press, Cambridge, England, 1990).

${ }^{15}$ A. H. Narten et al., J. Chem. Phys. 56, 1185 (1972).

${ }^{16}$ M. C. Bellissent-Funel, P. Chieux, D. Levesque, and J. J. Weis, Phys. Rev. A 39, 6310 (1989).

${ }^{17}$ X. G. Gong et al., Europhys. Lett. 21, 469 (1993).

${ }^{18}$ A. DiCicco and A. Filipponi, Europhys. Lett. 27, 407 (1994).

${ }^{19}$ E. D. Crozier and E. Murphy, Can. J. Phys. 50, 1914 (1972).

${ }^{20}$ H. Mayer and M. H. El Naby, Z. Phys. 174, 280 (1963).

${ }^{21}$ D. Nattland, H. Heyer, and W. Freyland, Z. Phys. Chem., Neue Folge 149, 1 (1986).

${ }^{22}$ B. R. Orton and S. P. Smith, Philos. Mag. 14, 873 (1966).

${ }^{23}$ H. Ocken and C. N. J. Wagner, Phys. Rev. 149, 122 (1966).

${ }^{24}$ L. G. Schulz, Adv. Phys. 6, 102 (1957).

${ }^{25}$ J. N. Hodgson, Adv. Phys. 16, 675 (1967).

${ }^{26}$ F. A. Richardson, Physical Chemistry of Melts in Metallurgy (Academic Press, London, 1974).

${ }^{27}$ M. J. Regan, H. Tostmann, P. S. Pershan, O. M. Magnussen, E. DiMasi, B. M. Ocko, and M. Deutsch, Phys. Rev. B 55, 10786 (1997).

${ }^{28}$ M. J. Regan, P. S. Pershan, O. M. Magnussen, B. M. Ocko, M. Deutsch, and L. E. Berman, Phys. Rev. B 55, 15874 (1997).

${ }^{29}$ G. C. Smith, J. Fischer, and V. Radeka, IEEE Trans. Nucl. Sci. NS-32, 521 (1985). The PSD was designed at Brookhaven National Laboratory (BNL) and consists of an $8 \mathrm{~cm}$ anode wire with $40 \mathrm{psi} \mathrm{Kr} / 10 \% \mathrm{CO}_{2}$ gas optimized for x-ray absorption at $19 \mathrm{keV}$.

${ }^{30} \mathrm{X}$-ray photoelectron spectroscopy (XPS) shows that no impurity segregates at the surface of liquid In contained in a Mo pan. The sample was heated up to $300{ }^{\circ} \mathrm{C}$ for several days and the XPS was taken after cooling the sample. The surface sensitivity of the XPS spectrometer is $1 \%$ given a penetration depth of about 30 $\AA$. This is in accordance with the In-Mo phase diagram which predicts no Mo solubility in In at these temperatures [T. B. Massalski, Binary Alloy Phase Diagram (ASM International, Materials Park, OH, 1990)].
${ }^{31}$ A. Roth, Vacuum Technology (Elsevier, Amsterdam, 1990).

${ }^{32}$ J. Fine, S. C. Hardy, and T. D. Andreadis, J. Vac. Sci. Technol. 18, 1310 (1981).

${ }^{33}$ JRS Scientific Instruments, Affoltern a.A., Switzerland.

${ }^{34}$ A. Guinier, X-Ray Diffraction (Freeman, San Francisco, 1963).

${ }^{35}$ A. Braslau, P. S. Pershan, G. Swislow, B. M. Ocko, and J. AlsNielsen, Phys. Rev. A 38, 2457 (1988).

${ }^{36}$ The microscopic definition of the local density profile $\widetilde{\rho}\left(z-h\left(\vec{r}_{x y}\right)\right)$ requires averaging over density fluctuations with wavelengths shorter than $\xi$. It follows that fluctuations with surface wave vectors $q_{x y} \leqslant \pi / \xi$ are contained in the definition of $\widetilde{\rho}\left(z-h\left(\vec{r}_{x y}\right)\right)$.

${ }^{37}$ S. K. Sinha, E. B. Sirota, S. Garoff, and H. B. Stanley, Phys. Rev. B 38, 2297 (1988).

${ }^{38}$ T. Iida and R. I. L. Guthrie, The Physical Properties of Liquid Metals (Clarendon Press, Oxford, 1993).

${ }^{39}$ This cutoff is selected such that the number of capillary modes is equal to the number of surface atoms. Choice of a larger cutoff length would imply that the local density profile includes contributions from averaging over the capillary waves with the shortest wavelengths. First principle arguments that might support such a larger cutoff length are beyond the scope of this paper. A smaller cutoff length on the other hand would imply capillary waves with wavelengths smaller than the particle size which is not physically reasonable.

${ }^{40}$ B. M. Ocko, X. Z. Wu, E. B. Sirota, S. K. Sinha, and M. Deutsch, Phys. Rev. Lett. 72, 242 (1994).

${ }^{41}$ M. P. Gelfand and M. E. Fisher, Physica A 166, 1 (1990).

${ }^{42}$ Experimentally determined data for the bulk structure factor $S(Q)$ and the pair correlation function $g(R)$ calculated from $S(Q)$ can be found in Y. Waseda, The Structure of NonCrystalline Materials (McGraw-Hill, New York, 1980); an electronic database can be found at http://www.iamp.tohoku.ac.jp/ database/scm/index.html

${ }^{43}$ O. Tomagnini, F. Ercolessi, S. Iarlori, F. D. Di Tolla, and E. Tosatti, Phys. Rev. Lett. 76, 1118 (1996).

${ }^{44}$ M. Fukuto, R. K. Heilmann, P. S. Pershan, J. A. Griffiths, S. M. $\mathrm{Yu}$, and D. A. Tirrell, Phys. Rev. Lett. (to be published).

${ }^{45}$ C. Gourier et al., Phys. Rev. Lett. 78, 3157 (1997).

${ }^{46}$ H. Tostmann, E. DiMasi, P. S. Pershan, B. M. Ocko, O. G. Shpyrko, and M. Deutsch, Ber. Bunsenges. Phys. Chem. 102, 1136 (1998). 